\title{
Elementos fundamentales para el cuidado en guarderías privadas cubanas: perspectiva desde la enfermería*
}

Fundamental elements for the care in Cuban private nurseries: a Nursing perspective Elementos fundamentais para o cuidado em creches privadas cubanas: perspectivas da enfermagem

\section{José Martín Medina Pérez ${ }^{\mathrm{I}}$ Julia Maricela Torres Esperón ${ }^{\mathrm{II}}$, Carilaudy Enriquez González ${ }^{\mathrm{III}}$}

Resumen: Objetivo: explorar experiencias, relacionadas con elementos fundamentales en el ejercicio de los cuidados en guarderías privadas por parte de las enfermeras trabajadoras en guarderías estatales. Método: estudio cualitativo de tipo exploratorio realizado en octubre de 2020, con 15 enfermeras que laboran en guarderías estatales del municipio Santa Clara, provincia Villa Clara, Cuba. Los datos se obtuvieron mediante dos grupos focales; las narrativas fueron procesadas mediante análisis de contenido. Resultados: emergieron cinco categorías: alimentación del niño, preparación para el cuidado, control de la salud de las cuidadoras, ambientes saludables para el cuidado y horarios de vida. Conclusiones: se destaca la preparación de alimentos y dieta, así como de cuidadoras que deberían recibir algún tipo de certificación y tecnologías para la capacitación; se recomienda la limpieza, ventilación, iluminación, observación de riesgos de accidentes; los horarios de vida y su vínculo con el desarrollo físico, psicomotor, social y afectivo de los infantes.

Descriptores: Cuidado del Niño; Guarderías; Niño; Enfermería; Enfermería en Salud Comunitaria

Abstract: Objective: to explore experiences related to fundamental elements for the care in private nurseries, carried out by nurses who work in public nurseries. Method: an exploratory qualitative study was carried out in October 2020, with 15 nurses who work in public nurseries from Santa Clara municipality, Villa Clara province, Cuba. The information was obtained using two focal groups; narratives were processed by means of content analysis. Results: five categories came up: child feeding, training for care, health control of carers, healthy environment for care and life schedule. Conclusion: food preparation and diet are highlighted, as well as the carers who should receive some kind of certificate and technologies for training. Cleaning, ventilation, illumination,

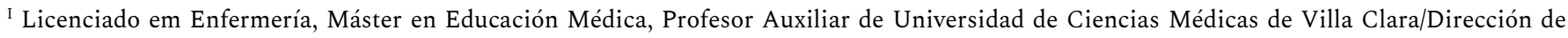
Ciencia y Técnica, Santa Clara, Cuba. E-mail: jmmperez@infomed.sld.cu, Orcid: https://orcid.org/0000-0003-0517-263X

II Licenciada em Enfermería, Doctor em Ciencias de la Salud. Profesor Titular de Escuela Nacional de Salud Pública, Departamento Dirección en Salud, La Habana, Cuba. E-mail: mtorresesperon7@gmail.com, Orcid: https://orcid.org/0000-0002-0702-7025

III Licenciada em Enfermería, Doctor en Ciencias de la Enfermería, Profesor Titular de Universidad de Ciencias Médicas de Villa Clara/Departamento de Ingreso y Formación Vocacional, Santa Clara, Cuba. E-mail: carilaudyeg@infomed.sld.cu, Orcid: https://orcid.org/0000-0001-5443-4865
}

*Extraído de una investigación de tesis doctoral "Intervención de enfermería para el fomento de la salud infantil en instituciones no estatales de cuidados a infantes sanos. Santa Clara. 2019-2022”. Programa de Posgrado em Enfermería, Universidad de Ciencias Médicas de La Habana, ano 2021. 
Elementos fundamentales para el cuidado en guarderías privadas cubanas: perspectiva... $\mid 2$

observation of accident risks; life schedule related to physical, psychomotor, social and emotional development of children are recommended.

Descriptors: Child Care; Schools, Nursery; Child; Nursing; Community Health Nursing

Resumo: Objetivo: explorar experiências relacionadas com elementos fundamentais no exercício dos cuidados desenvolvidos em creches privadas por enfermeiras trabalhadoras em creches estaduais. Método: estudo qualitativo do tipo exploratório realizado em outubro de 2020, com 15 enfermeiras que trabalham em creches estaduais do município de Santa Clara, província Villa Clara, Cuba. Os dados foram obtidos por meio de grupos focais; as narrativas foram tratadas mediante análise de conteúdo. Resultados: emergiram cinco categorias: alimentação da criança, preparação para o cuidado, controle da saúde das cuidadoras, ambientes saudáveis para o cuidado e rotina. Conclusões: destaca-se a preparação de alimentos e dieta, assim como de cuidadoras que deveriam receber algum tipo de certificação e tecnologia para a capacitação; recomenda-se a limpeza, ventilação, iluminação, observação de riscos para acidentes; a rotina e seu vínculo com o desenvolvimento físico, psicomotor, social e afetivo das crianças. Descritores: Cuidado da Criança; Creches; Criança; Enfermagem; Enfermagem em Saúde Comunitária

\section{Introducción}

La etapa preescolar en la infancia es considerada como una de las más importantes en la vida de un ser humano. Su desenvolvimiento en instituciones de cuidados de niños sanos es una variante de socialización de la infancia para el desarrollo, que además proporciona a las familias y fundamentalmente a las madres, la posibilidad de dar continuidad a la vida laboral. ${ }^{1}$

Según una revisión sistemática sobre guarderías en un centro para niños menores de cinco años en países de ingresos bajos y medianos se sugiere que el cuidado ofrecido en guarderías puede tener un efecto positivo en la capacidad cognitiva de los niños menores de cinco años de edad. ${ }^{2}$ Así mismo, otra investigación que indagó el desarrollo de niños en guarderías concluyó que éstas en niños muy pequeños puede ser positiva por la asociación con resultados psicosociales, así como en la motricidad fina y gruesa. ${ }^{3}$

Las instituciones de cuidados de niños sanos se han desarrollado a la par de la incorporación de las mujeres a la vida laboral activa. En algunos países se establecen dentro de las propias locaciones laborales y en otros en la comunidad. Son nombradas de diferentes maneras, sin embargo, la más utilizada en la literatura es "guardería”. 3 
Son múltiples los beneficios que sobre la salud de los infantes tiene la labor del personal de enfermería de Atención Primaria en las instituciones infantiles, que incluye no solo atender las necesidades básicas de salud del niño sino también promocionar su crecimiento y estimular su desarrollo. La utilización de los conocimientos, habilidades y valores propios de la profesión de enfermería en función de la asistencia ofrecida al niño, la familia y otros profesionales que trabajan en estos centros tienen un gran alcance. ${ }^{4}$

Una revisión integradora de la literatura, donde se seleccionaron 14 estudios completos para su análisis interpretativo concluyó que el trabajo del personal de enfermería es significativo para hacer factibles acciones de salud efectivas que aseguren mejor calidad de vida para los niños, promuevan su salud y desarrollo armonioso en el marco de la Atención Primaria. A pesar de que el cuidado infantil se considera actualmente un área multi- profesional de trabajo se reconoce la contribución del personal de enfermería a esta práctica por su dedicación a acciones de promoción de la salud, lo que favorece, en ocasiones, una mayor satisfacción del usuario. ${ }^{5}$

En el caso de Cuba a estas instituciones se les llama círculos infantiles o jardines de infancia, las cuales fueron creadas en todo el país a partir de la década de los 1960 y desde ese momento se concibieron con la inclusión del personal de enfermería de manera permanente. Este es entrenado para la vigilancia sanitaria, cuidados a infantes sanos y la asesoría en el desarrollo infantil. Es práctica habitual la estabilidad laboral de las enfermeras en dichas instituciones, por lo que muchas de ellas son referentes en la labor que realizan. ${ }^{1}$

A partir de la implementación en este siglo de un nuevo modelo económico en el país se generó una nueva modalidad de guarderías, en este caso "guarderías infantiles privadas”. Con ellas aparece como forma de empleo la "actividad para la educación y el cuidado de niños”. Plaza que pueden desempeñar personas dueñas de guarderías u otras que son empleadas por estas, las cuales deben cumplir como requisito realizarse el cheque médico preempleo, desde el punto de 
Elementos fundamentales para el cuidado en guarderías privadas cubanas: perspectiva... $\mid 4$

vista profesional no se exigen requisitos. Por lo que pueden ser desde amas de casa, técnicas o profesionales de diferentes ramas. ${ }^{6}$

Estas instituciones pertenecen al primer nivel de atención y responden a uno de los programas priorizados del Sistema Nacional de Salud, en este caso el Programa Nacional de Atención Materno Infantil, el que exhibe resultados exitosos en cuanto a indicadores sobre todo en el grupo de edad menor de seis años. ${ }^{7}$ Las trasformaciones y contextualización de este programa a las realidades histórico-sociales han sido una las fortalezas para estos éxitos, entre otras. En tal sentido en un artículo publicado sobre los logros y desafíos de la Atención a la salud materno infantil en Cuba se plantea: "Las estrategias, las normativas y los programas establecidos en beneficio de la salud materno - infantil requieren de una constante renovación, con la introducción de los nuevos conocimientos y tecnologías, sobre la base de las evidencias científicamente probadas; de ahí la importancia de las investigaciones y la aplicación de sus resultados”. 8

Es por ello que la existencia de guarderías privadas constituye un desafío para mantener y mejorar los indicadores de salud alcanzados por el Programa Nacional de Atención Materno Infantil. No obstante, la experiencia acumulada por el personal de enfermería en el cuidado en las guarderías estatales puede aportar de forma significativa para la articulación del programa a las nuevas condiciones y por ende a la calidad del cuidado en las guarderías privadas. De ahí que el problema de investigación planteado sea ¿explorar las experiencias de enfermeras que laboran en guarderías estatales acerca de los elementos fundamentales que deben ser considerados para ejercer los cuidados en las guarderías privadas cubanas? En correspondencia el objetivo de este artículo es explorar experiencias de enfermeras que laboran en guarderías estatales acerca de los elementos fundamentales que deben ser considerados para ejercer los cuidados en las guarderías privadas cubanas. 


\section{Método}

Se realizó un estudio cualitativo con diseño exploratorio, cuya población de estudio fueron enfermeras que laboran en guarderías estatales, llamadas en el país como círculos infantiles o jardines de la infancia. Actualmente en el municipio de Santa Clara de la provincia de Villa Clara existen 32 instituciones de este tipo, en las que se desempeña una enfermera en cada una.

En las guarderías estatales se organizan los niños y niñas por años de vida, se establecen en cada salón grupos de hasta 20 infantes de ambos sexos, en cada uno se desempeña una cuidadora y una auxiliar. Cada institución tiene una enfermera que se ocupa del control de los cuidados y de la salud de infantes y trabajadoras. Para la selección de las enfermeras participantes se consultó con las supervisoras de Enfermería del municipio para identificar las características de este personal e identificar los criterios homogeneidad, dado que éste fue el tipo de muestreo empleado, dichos criterios fueron: Poseer cinco o más años de trabajo en guarderías estatales y evaluaciones satisfactorias de su proceso de trabajo durante ese tiempo. Dado que las enfermeras en el país poseen en su expediente laboral los resultados de sus evaluaciones anuales. De manera que participaron 15 enfermeras que cumplían los criterios de selección, las cuales fueron contatadas de manera personal y de todas se obtuvo la aprobación para participar.

Para la organización del estudio las participantes se dividieron en dos grupos, uno con siete enfermeras que tenían entre 5 a 10 años de experiencia y otro con ocho con más de 10 años de experiencia. Para la recogida de datos se seleccionó la técnica de grupo focal. Dicha elección se justifica porque esta técnica permite sustentar el ideario grupal sobre una temática o hecho social que es objeto de investigación. ${ }^{9}$

El 25 de octubre de 2020 se realizaron dos grupos focales de aproximadamente 45 minutos cada uno con la pregunta disparadora que fue: ¿Qué elementos deben ser considerados para el fomento del cuidado de la salud infantil en las guarderías privadas? Dicha pregunta se elaboró de manera empírica a partir del problema científico que generó el estudio. Cada grupo focal se 
Elementos fundamentales para el cuidado en guarderías privadas cubanas: perspectiva... $\mid 6$

materializó individualmente en el aula de post-grado del edificio de la carrera de Enfermería de la Universidad de Ciencias Médicas de Villa Clara. Cada sesión fue grabada por dos teléfonos móviles y el debate fue guiado por una moderadora que fungió como investigadora alterna; el investigador principal se desempeñó como observador y otras dos investigadoras participaron como auxiliares de la investigación.

Con anterioridad se hizo un esquema de la posición de los asientos que se colocaron de forma circular. Las dos sesiones de grupos focales transcurrieron una a continuación de la otra; en cada caso la moderadora explicó las características de la actividad a realizar, así como la importancia del diálogo y al respeto de cada opinión. Estos elementos y la pregunta estuvieron visibles en una pancarta durante toda la sesión. La conversación se desarrolló espontáneamente, con respeto a los criterios individuales e intercambios de profundización de la moderadora para cerrar los diferentes temas emergentes. Oportunidad que se fue aprovechando para el feedback sobre los resultados.

Las narrativas de las sesiones fueron grabadas, transcritas y agrupadas en un cuerpo textual. Las notas de campo fueron hechas por los observadores durante las sesiones, no obstante, para el análisis que se presenta en este artículo, solo se utilizaron las narrativas grabadas y transcritas. Para garantizar el anonimato de las enfermeras participantes se les asignó un código para el registro $(\mathrm{E})$ y (Ea) para el segundo grupo y se enumeraron de acuerdo al orden con que hicieron uso de la palabra. Para el procesamiento de la información se utilizó la técnica de análisis de contenido de acuerdo a los criterios de Bardin, ${ }^{10}$ en el que se empleó un análisis temático para obtener categorías empíricas. Dicho proceso fue estructurado en tres fases: 1) pre-análisis, 2) exploración del material y 3) tratamiento de resultados, inferencia e interpretación. La misma se realizó de manera manual por dos de los autores independiente y posteriormente se consensuó el resultado final.

En la fase de pre-análisis se organizó el cuerpo textual y se realizó la lectura fluctuante con el propósito de apropiarse de las ideas de inicio. Se decidió utilizar el método colorimétrico 
para seleccionar las unidades de registro. En la fase de exploración del material fue realizada la lectura en profundidad del cuerpo textual, los datos ordinarios fueron transformados, organizados y reunidos en unidades que permitieron la descripción de las características pertinentes del contenido, se identificó con diferentes colores las unidades de registro que emergieron y se buscaron los núcleos de sentido que dieron lugar a las categorías. En la fase de tratamiento de resultados, inferencia e interpretación se ejecutó el análisis categorial, las que emergieron del análisis y se aplicó la frecuencia ponderada que posibilitó comprobar el peso de frecuencia de cada categoría.

La investigación fue evaluada y aprobada por los siguientes acuerdos de la Facultad de Enfermería y Tecnología de la Salud de la Universidad de Ciencias Médicas de Villa Clara: 85/ 2019 del Consejo Científico; 13/ 2020 del Comité de ética de la investigación científica y 9/2020 del Consejo de dirección. Para el proceso investigativo se ha respetado lo acordado por la Asociación Médica Mundial y que aparece en la Declaración de Helsinki. ${ }^{11}$ Las participantes firmaron un documento que expresa su consentimiento informado sobre su aceptación en el desarrollo de la investigación. Las transcripciones fueron devueltas a las participantes para comentarios y corrección después de concluida esa fase.

\section{Resultados}

Las participantes todas son del sexo femenino con un promedio de edad 46 años, licenciadas en enfermería con más de 20 años en promedio como profesionales y más de cinco años en guarderías estatales, de las cuales todas han recibido entrenamiento para su función y dos poseen maestría. Durante el procesamiento de la información emanada de los dos grupos focales emergieron cinco categorías: alimentación del niño, preparación para el cuidado, control de la salud de las cuidadoras, ambientes saludables para el cuidado y horarios de vida. 
Elementos fundamentales para el cuidado en guarderías privadas cubanas: perspectiva... $\mid 8$

Dada la experiencia de las enfermeras participantes estas categorías pueden considerarse aspectos fundamentales para el cuidado en guarderías privadas, considerando que dichas guarderías son alternativas nuevas y sus cuidadoras pueden ser personas de diferentes profesiones con licencias otorgadas por las instancias de educación y contratadas por las dueñas, en algunos casos son las propias dueñas las cuidadoras. A seguir se describen las categorías según el peso obtenido por la frecuencia ponderada.

Categoría 1: alimentación del niño. Esta categoría emerge del ideario profesional de las enfermeras y los resultados se enfocan básicamente en el aspecto de elaborar los alimentos en las guarderías privadas para garantizar las normas de higiene y el control, y en la dieta que se debe conformar para garantizar una nutrición adecuada. En este caso la experiencia vital de las participantes es esencial para comprender el fenómeno de la importancia de la elaboración de los alimentos y apunta a la garantía de la higiene y a evitar intoxicaciones alimentares. Esta categoría le da una importancia especial a la nutrición adecuada y al manejo de los trastornos gastrointestinales y patologías que requieran la ingestión de dietas terapéuticas. Los discursos que justifican esta categoría se presentan a continuación

es muy importante la elaboración de alimentos.(E6)

se debe cocinar junto para todos.(E5)

es mejor que la comida de los niños se elabore allí y que se recojan muestras testigos.(E1)

respetar la higiene de los alimentos y respetar las normas de la conservación de los alimentos.(E8)

la confección de la dieta.(E7)

siempre hay que tener en cuenta los horarios de merienda, los grupos alimentares, cuales se pueden dar para evitar obesos y desnutridos.(E7) tener en cuenta a los intolerantes a la lactosa.(E2a) se sientan distintos al momento de alimentarse, de acuerdo a las patologías.(E5a) 
Categoría 2: preparación para el cuidado. Esta categoría sienta la necesidad de formación de las cuidadoras y a los mecanismos que permitirían lograrla. Se refiere esencialmente al fenómeno formativo y educativo que debe involucrar a la persona para convertirla en cuidadora de guarderías. Apunta a la necesidad de la existencia de una tecnología educativa previa que ayude a la formación de los cuidadores de las guarderías y a su actualización sistemática. Los discursos a continuación permitieron la categoría emergente:

las cuidadoras deben recibir una capacitación. (E1) un curso de capacitación, que tengan un libro, un cuaderno; puede ser un folleto que se actualice cada cierto tiempo, deben tener preparación, educación, lenguaje adecuado. (E3)

la promotora del programa "Educa a tu hijo" las puede ayudar para los roles de juego, [...] por ejemplo existen cursos de un mes para auxiliares pedagógicas, en este caso puede ser así. (E6a) un manual, un texto básico sobre cómo cuidar niños en una casa. (E6) elaborar un manual que se vaya actualizando. (E4a) la parte educativa de las cuidadoras es esencial. (E6a) para reconocer al niño sano con respecto al niño con patologías. (E3a) ellas deben ser evaluadas y si no tienen condiciones retirarle las licencias. (E8)

Categoría 3: control de la salud de las cuidadoras. En esta categoría se manifiestan en dos aspectos esenciales, los que se dirigen la importancia de la salud de las cuidadoras y su implicación para la protección de la salud de los niños. Los discursos que respaldan esta categoría se presentan a continuación.

las cuidadoras deben tener actualizado su carné de salud. Es necesario velar también por su salud. (E1) se debe saber quiénes son esas personas, sus antecedentes. (E7) y ser supervisadas cada cierto tiempo por el área de salud. (E6a) deberían ser evaluadas por alguna autoridad al menos una vez al mes. (E7a) 
Elementos fundamentales para el cuidado en guarderías privadas cubanas: perspectiva... | 10

Categoría 4: ambientes saludables para el cuidado. En esta categoría se muestra el valor que ellas le confieren al espacio, la ventilación, la iluminación, la higiene y la minimización de los peligros en las guarderías. Las voces que respaldan este resultado se colocan a seguir.

Las casas deben tener buenas condiciones higiénicas y confortables, no deben existir peligros. (E3)

amplias, ventiladas, bien iluminadas y con mucha higiene. (E4)

la higiene de esas casas debe ser tres veces por día. (E6a)

Categoría 5: horarios de vida. Este aspecto es también de importancia para las enfermeras que participan en el estudio, dado que enfatizan en el valor del horario de sueño, de alimentación y de juegos, como se puede observar en las voces a seguir:

se debe respetar los horarios de vida de los niños, sobre todo el sueño. (E1) es indispensable tener previsto el horario de sueño. (E7) respetar los horarios de alimentación, de juego, de dormir. (E4a)

\section{Discusión}

En los resultados de este estudio emerge la alimentación del niño como una necesidad humana esencial y considerada uno de los ejes temáticos de la práctica desde la mayoría de las escuelas teóricas y programas de formación de enfermería. Los autores de la investigación, desde su experiencia profesional han vivenciado la asignación de funciones al personal de enfermería en la práctica asistencial en círculos infantiles, donde supervisan el proceso de elaboración de los alimentos, controlan las normas de higiene establecidas, así como las condiciones higiénicas de los utensilios, asesora al personal responsabilizado (cocineros y auxiliares de cocina) y verifica que el cumplimiento de la toma de muestras testigo de alimentos se realice de acuerdo a las normas establecidas. En caso de presentarse cualquier intoxicación alimentaria son las encargadas de comunicar de inmediato esta situación a las autoridades sanitarias del Policlínico. 
Con respecto a la categoría "alimentación del niño", en lo relacionado a la elaboración de los alimentos se comprende el interés por el procedimiento y manipulación en su confección, la cual debe realizarse de manera integral y única a nivel de la guardería como elemento que garantiza su higiene y que evitaría la aparición de intoxicaciones alimentarias y el parasitismo intestinal. Éste última causa común en instituciones de socialización de niños pequeños, como reflejan algunos estudios donde la prevalencia de giardia lamblia y la ento ameba coli en niños de ambos sexos usuarios de guarderías fue elevada. ${ }^{12-13}$ Así como en la asociación entre el comprometimiento del crecimiento del peso y la estatura en niños de ambos sexos que eran cuidados en guarderías y fueron hospitalizados por causas relacionadas con el parasitismo. ${ }^{14}$

La calidad de los alimentos es otro factor fundamental para la salud de los niños, según el parecer de las enfermeras. En tal sentido los manipuladores de alimentos son los principales responsables de la prevención y el control de la contaminación de los alimentos. Estudios publicados reflejan resultados de investigaciones realizadas en guarderías que revelan el requerimiento de la higiene personal, uso de ropa adecuada, como medios de protección como el naso buco y el gorro y el lavado de manos de acuerdo a los protocolos establecidos. ${ }^{15}$

Con respecto a la administración de la dieta parece tener su fundamento en los conocimientos de nutrición y dietética que forman parte del acervo formativo del personal de enfermería, lo que les permite convertirse en promotor de la salud nutricional de la población y vigilar desviaciones que pueden conducir a la malnutrición. Así como de la experiencia vital en sus lugares de labor donde participan de conjunto con el personal administrativo en la confección del menú y control de la dieta que se oferta a los educandos, de forma que se garantice su aporte calórico, con la valoración de las dietas especiales y de infantes intolerantes a los diferentes alimentos. ${ }^{1,4}$

En este sentido se pueden mencionar los resultados de dos estudios realizados en 25 guarderías donde se encontraron problemas de malnutrición en niños que asistían a las mismas. ${ }^{16-17}$ Es por ello que mantener la vigilia en este aspecto es esencial. Dada la relación que tiene 
Elementos fundamentales para el cuidado en guarderías privadas cubanas: perspectiva... | 12

la malnutrición con el rendimiento académico. La malnutrición es uno de los problemas que más afecta a la población infantil de los países en vías de desarrollo. El término "malnutrición” engloba estados nutricionales, como la desnutrición y la obesidad. Un estado de nutrición óptima asegura a un niño con menos problemas de salud y con más facilidades en el aprendizaje. ${ }^{18}$

En este tema también se identificó una revisión sistemática que incluye 303 estudios, que identifican cuatro líneas de acción para la promoción de las condiciones de higiene y de la inocuidad de los alimentos como temas fundamentales para el cuidado de la salud en los centros de cuidado infantil; los aspectos más relevantes de estas líneas se dirigen a: lavado de manos, ventilación, manejo de residuales líquidos y manipulación de alimentos. ${ }^{15}$ Todo hace coherente el resultado de la categoría alimentación del niño, como la de mayor peso en este estudio.

A la luz de los resultados de este artículo se reconoció interesante también, un estudio en el que se evaluó la planificación y organización de la nutrición en guarderías públicas y privadas, como resultado se registra que, a pesar de las tasas nutricionales más altas en las guarderías no públicas, se observaron algunos errores en la planificación de la nutrición. La mayor cantidad promedio de dinero asignada por día para alimentar a un niño en guarderías no públicas no proporcionó una nutrición adecuada. Se recomienda que es necesario publicar recomendaciones estandarizadas, comprensibles y prácticas sobre la nutrición de los niños en las guarderías. La adopción de tales recomendaciones en la práctica diaria en todas las guarderías públicas y privadas debería mejorar la nutrición de los niños. ${ }^{19}$

Al parecer esta categoría tiene su origen en los valores individuales, sociales y profesionales de las enfermeras participantes. Ya desde el enfoque de la fundadora de la enfermería moderna, el entorno era esencial para la salud de las personas ${ }^{20}$ y esta concepción se ha trasmitido durante generaciones de enfermeras convirtiéndose en parte de sus valores y su práctica. A la vez que ha evolucionado con los nuevos conocimientos científicos y con la asunción de otras teorías que también se refieren al ambiente. A su vez la propia práctica 
profesional aporta a este ideario dadas las funciones de este personal como encargado de revisar los locales relacionados con el proceso de alimentación: cocina, pantrys, almacén de alimentos y comedores. Así como las áreas internas: salones de estancia, servicios sanitarios de los educandos y de los trabajadores y las áreas externas: cisternas (con suficiente agua para el día), peligros potenciales con riesgos de accidentes.

La segunda categoría: preparación para el cuidado, se percibe sustentada en la experiencia vivencial del personal de enfermería, que ha debido cumplir el requisito social de la formación para poder ejercer el cuidado de manera profesional; a la vez que identifica escenarios y actores de su propia práctica como educadoras y promotoras de salud. Desde la perspectiva de las enfermeras, para que se ejerza un cuidado particular como es el cuidado grupal de los infantes es necesario que las cuidadoras tengan acceso al desarrollo de capacidades para el ejercicio de esta actividad (el cuidado).

La educación debe encargarse de desarrollar las capacidades intelectuales, emocionales/afectivas, físicas, actitudinales/axiológicas y conductuales/comportamentales de sus alumnos. Todo ello, con la finalidad de formar buenos ciudadanos que den respuesta a un mundo global y complejo con base en los parámetros del respeto de los derechos humanos y en busca de la justicia social. ${ }^{21}$

Son escasas las publicaciones que tratan el tema de la capacitación de los cuidadores que laboran en guarderías, no obstante, a los autores del presente artículo llamó la atención uno dónde se evidencian los resultados de un programa de guarderías y estancias infantiles para apoyar a madres trabajadoras. El programa ofrecía cursos, talleres y demás actividades de capacitación a las personas interesadas en establecer y operar una guardería y estancia infantil. ${ }^{22}$

Un elemento que es mencionado por las enfermeras en esta categoría es la promotora del programa "Educa a tu hijo" que recomienda su ayuda para los roles de juego. Este programa es "La modalidad no institucional para la atención educativa a los niños de la primera infancia. 
Elementos fundamentales para el cuidado en guarderías privadas cubanas: perspectiva... | 14

Está dirigido a preparar a las familias para qué a partir de sus propias experiencias y saberes, realicen acciones educativas con sus hijas e hijos desde las condiciones del hogar”. ${ }^{23}$ La idea de trabajar de conjunto con el programa es loable, dado que el mismo está consolidado en las comunidades y las promotoras son personas preparadas para sus funciones.

Otro aspecto interesante es que las enfermeras participantes proponen como un ejemplo de capacitación que pudieras recibir las cuidadoras de guarderías privadas, los cursos que se les ofrece a las auxiliares pedagógicas que trabajan en el sector estatal. Esta alternativa puede ser aceptada por los gestores de educación que organizan dichas capacitaciones, dado que los mismos se ofrecen a nivel de municipios.

En esta categoría también emerge la propuesta de un manual para la capacitación de las cuidadoras, a partir de la experiencia de las enfermeras que en su preparación utilizan textos de formatos diversos para su propia formación como cuidadoras. Así como en su acervo profesional, donde el uso de manuales y folletos para la educación sanitaria constituyen tecnologías habituales en su práctica educativa con padres y empleados de las guarderías estatales. Este aspecto es de gran valor para el proyecto donde están inmersos los resultados de este artículo, dado que entre los objetivos está el diseño de tecnologías educativas para las cuidadoras de guarderías privadas.

Las tecnologías educativas se conciben desde dos dimensiones, como procesos y como productos. ${ }^{24}$ Como proceso pueden orientar los procedimientos establecidos para el diseño y como producto ofrecen variadas alternativas en soporte digital o formato impreso. ${ }^{24-25}$ Todas ellas, con una concepción adecuada y contextualizada a las necesidades educativas, enriquecidas por la experiencia práctica de enfermería, pueden contribuir a la reflexión y modificación de modos de actuación a favor de la prevención y promoción de salud. ${ }^{25}$

La tercera categoría sobre control de la salud de las cuidadoras emerge ciertamente de la vivencia práctica de las enfermeras, ya que son ellas el personal al que se le ha otorgado el mayor 
peso profesional en el control sanitario que se exige a los empleados de las guarderías estatales. El objetivo es garantizar la salud de las trabajadoras y en consecuencia proteger la salud de los niños con los que están en contacto.

Los trabajadores de las guarderías estatales en Cuba, están sujetos a controles sanitarios sistemáticos, que no se establecen para los trabajadores de las guarderías privadas; a pesar de que el propio decreto-ley número 356 sobre el ejercicio del trabajo por cuenta propia en su Capítulo II faculta al Ministerio de Salud Pública: en el control y la vigilancia sanitaria y epidemiológica, otorgar la licencia sanitaria y establecer las regulaciones para el cuidado de niños, enfermos, personas con discapacidad y ancianos. ${ }^{6}$ De ahí que este resultado, es una alerta dentro del estudio para el trabajo intersectorial que permitan lograr los controles sanitarios establecidos y con ello preservar la salud de infantes de guarderías privadas.

Las capacidades personales previas que debe demostrar una persona que vaya a dedicarse al cuidado en guarderías no tienen mucho tratamiento en la literatura especializada. Sin embargo, en el ya citado artículo que aborda el Programa de guarderías y estancias infantiles para apoyar a madres trabajadoras, se menciona como las personas interesadas en establecer y operar una guardería y estancia infantil en México estaban obligadas a demostrar capacidad o experiencia para ofrecer servicios de cuidado infantil, alimentación y actividades lúdicas. ${ }^{23}$

Por otro lado los resultados de un estudio dirigido a analizar la relación entre las competencias y habilidades requeridas en el puesto, con el nivel de burnout experimentado por personal de estancias infantiles y que incluyó a 58 empleadas de tres estancias infantiles privadas, sugieren que dominar ciertas competencias o habilidades requeridas para un adecuado cuidado infantil, en especial la de establecer vínculos afectivos, constituye un factor preventivo para el desarrollo del síndrome de burnout en personas que se desempeñan en estancias infantiles; y que, por el contrario carecer de éstas, constituye una condición de vulnerabilidad para el desarrollo del mismo. ${ }^{26}$ Es por tanto la salud de las cuidadoras un elemento que también 
Elementos fundamentales para el cuidado en guarderías privadas cubanas: perspectiva... | 16

se debe estudiar para prevenir afecciones de disimiles tipos, lo que es también una garantía de mejores cuidados para los infantes.

Asimismo, es importante destacar que el control encuentra alguna evidencia en el propio decreto-ley número 357 el cual en su capítulo I define contravenciones y medidas aplicables a los infractores del trabajo por cuenta propia (designación a trabajadores del sector privado en el país). ${ }^{6}$ Este es un elemento a valorar en el estudio que da lugar al artículo, dado que en otra fase del mismo se debe explorar con cuidadoras del sector privado sobre su preparación para las funciones que ejercen.

La categoría relacionada con ambientes saludables para el cuidado es también un factor esencial para el desarrollo infantil. Aspecto igualmente relacionado para enfermería con sus bases teóricas desde Florence Nightingale vigente en la actualidad. ${ }^{20}$ La visión que se obtiene de estos resultados a los efectos del presente artículo es la asunción de esta línea como premisa esencial para el funcionamiento efectivo de las guarderías traducido a la salud infantil.

En tal sentido el Plan Estratégico 2018-2021 del Fondo de las Naciones Unidas para la Infancia (UNICEF) declara en el cuarto objetivos "cada niño vive en un ambiente seguro y limpio comprende la esfera del agua, el saneamiento y la higiene, así como las iniciativas relacionadas con el clima, la reducción del riesgo de desastres y los entornos urbanos. ${ }^{27}$ Estos elementos deben tenerse en cuenta en cualquier ambiente en se desarrolle un niño, entre ellos, las guarderías deben constituir prioridad, ya sean públicas o privadas.

Al respecto también se han realizado investigaciones sobre las condiciones estructurales de las guarderías y el parasitismo como factores influyentes en el estado nutricional de micronutrientes de los niños en un estudio donde se midieron las concentraciones de hemoglobina, de zinc y de retinol en 271 niños que asistían a guarderías. En el mismo se comenta que guarderías de baja calidad, frecuentadas por niños de mayor vulnerabilidad, implicarían dificultades institucionales de cumplir su deber de promover a salud. ${ }^{17}$ 
Los autores del presente artículo destacan la responsabilidad de las cuidadoras al identificar las condiciones socioeconómicas de las familias de los niños que asisten a su guardería, para minimizar en lo posible sus efectos sobre la nutrición. Asimismo, velar por las condiciones estructurales e higiénicas que puedan favorecer la ocurrencia de lesiones no intencionales.

Finalmente, la categoría horarios de vida, que no por ser la quinta en el orden del peso en que emergieron, sea menos importante. Ella debe sustentarse también en la experiencia práctica de las enfermeras que laboran en guardería estatales, donde se materializan procesos de educación y cuidado de niños. En la conceptualización de la higiene pedagógica el respeto de los horarios de vida es esencial para el alcance de un desarrollo psicopedagógico óptimo.

Entre los problemas relacionados con los horarios de vida, uno de los que puede ocasionar trastornos en los niños son los relacionados con el sueño, en tal sentido en una revisión integradora se refieren entre los estudios citados a uno en que se encontró una prevalencia de problemas del sueño en el 38\% de los niños que asistían a guarderías y también mencionan un estudio que incluyó a 107 niños en edad pre - escolar donde se evidencia que a través de la utilización de una guía se identificó que el $75.7 \%$ de los niños que asistían a guarderías tenían problemas relacionados con el sueño. ${ }^{28}$ Estos resultados alertan al valor de los horarios de sueño en estas instituciones.

El sueño en la infancia es fundamental debido a que mientras se duerme ocurren diversos procesos de maduración y desarrollo del sistema nervioso, como la liberación de la hormona del crecimiento, la consolidación de la memoria, la regulación metabólica y el restablecimiento de la energía. El sueño adecuado conlleva a mejor rendimiento en el aprendizaje, la capacidad de focalizar la atención y la consolidación de la memoria, mejora las estrategias cognitivas y la salud de los niños escolares. Si se establecen hábitos de sueño adecuados en etapas tempranas es probable que permanezcan hasta la edad adulta, por ello es importante dar a conocer la relevancia del sueño en el desarrollo de los niños y promover los hábitos saludables con los 
Elementos fundamentales para el cuidado en guarderías privadas cubanas: perspectiva... | 18

padres, maestros y especialistas de la salud, ya que varios de los problemas de sueño pasan desapercibidos o no se les da importancia. ${ }^{29}$

Otro aspecto importante es el juego y los tiempos dedicados al mismo, al respecto un estudio realizado en 15 guarderías de la Asociación Recreativa y Cultural “Turma da Touca”, localizada en la zona sur del municipio de São Paulo y que involucró 285 niños entre 3 a 3 años y 11 meses, revelan la integración entre el juego y el aprendizaje con una comunicación oral efectiva. ${ }^{30}$

Los autores identifican en las participantes, que sus discursos están signados por sus experiencias personales, profesionales y formativas en la aspiración de nivelar las características del cuidado que se ofrece en las guarderías privadas al cuidado profesional que ellas brindan habitualmente en el ámbito de las guarderías estatales. Su experiencia es de gran valor para la toma de decisiones dentro del estudio.

\section{Conclusión}

Las cinco categorías que emergieron de este estudio ofrecen una visión general de los elementos fundamentales que deben ser considerados para ejercer los cuidados en las guarderías privadas cubanas. La experiencia vital y profesional de las enfermeras participantes se enfocan en la importancia de la alimentación de los niños, con énfasis en la preparación de los alimentos y en la dieta a administrar; la preparación de las cuidadoras, que se dirige a la posibilidad de recibir algún tipo de certificación y a la existencia de tecnologías para la capacitación; a la necesidad de garantizar ambientes saludables para el cuidado que incluye lugares amplios, ventilados, bien iluminados y limpios, donde se minimicen los riesgos de accidentes; insisten en el respeto a los horarios de vida y su vínculo con el desarrollo físico, psicomotor, social y afectivo de los niños; así como el control que garantiza que sean las personas adecuadas quienes ejerzan el cuidado y que su salud sea considerada y tratada por el sistema de salud. 
Los resultados de este estudio poseen aportes a la práctica, por su primicia en la necesidad de utilizar los saberes de enfermeras con experiencia en una labor tan importante como es el cuidado en instituciones que trabajan con la guarda de niños sanos. En tal sentido los resultados contribuyen a ofrecer una visión sobre las áreas de atención para el cuidado en guarderías privadas en Cuba, lo cual visto desde una perspectiva profesional de enfermería es de interés proteger a infantes y con ello mantener los indicadores de salud infantil alcanzados en el país.

\section{Referencias}

1. Franco García O. Los círculos infantiles en Cuba: una obra de la revolución. Laplage Rev. 2017;3(1):117-26. doi: 10.24115/S2446-6220201731240p.117-126

2. Brown TW, Van Urk FC, Waller R, Mayo-Wilson E. Centre-based day care for children younger than five years of age in low - and middle- income countries. Cochrane Database Syst Rev. 2014;(9):CD010543. doi: 10.1002/14651858.CD010543.pub2

3. Nair D, Alonge O, Hamadani JD, Salam SS, Islam I, Hyder AA. Developmental assessments during injury research: is enrollment of very young children in crèches associated with better scores. Int $\mathrm{J}$ Environ Res Public Health. 2017;14(10):1130. doi: 10.3390/ijerph14101130

4. Garcia Galvão DMP. La enfermera en guarderías/jardines de infancia: perspectiva de los profesores de una escuela superior de enfermería. Enferm Glob. 2018;17(51):368-80. doi: 10.6018/eglobal.17.3.291371

5. Goés FGB, Silva MA, Paula GK, Oliveira LPM, Mello NC, Silveira SSD. Nurses' contributions to goodpractices in childcare: an integrative literature review. Rev Bras Enferm. 2018;71(Suppl 6):2808-17. doi: 10.1590/0034-7167-2018-0416

6. Gaceta Oficial de la República de Cuba. Gaceta Oficial, 35 Extraordinaria [Internet]. La Habana: Ministerio de Justicia, 10 de julio de 2018 [citado 2019 oct 20]. Disponible en: http://www.fgr.gob.cu/sites/default/files/20180710/Publicaciones/goc-2018-ex35.pdf

7. Ministerio de Salud Pública (Cuba). Anuario estadístico de salud 2019 [Internet]. La Habana: Ministerio de Salud Pública de la República de Cuba; 2020 [citado 20 oct 2019]. Disponible en: https://www.researchgate.net/profile/Patricia-Alonso-

Galban/publication/341359801_Anuario_estadistico_de_Salud_2019/links/5ebc83e892851c11a86773b2/Anuarioestadistico-de-Salud-2019.pdf

8. Santana Espinosa MC, Esquivel Lauzurique M, Herrera Alcázar VR, Castro Pacheco BL, Machado Lubián MDC, Cintra Cala D, et al. Atención a la salud materno infantil en Cuba: logros y desafíos. Rev Panam Salud Pública [Internet]. 2018 [citado 2019 oct 20];42(27):1-10. Disponible en: https://www.scielosp.org/pdf/rpsp/2018.v42/e27/es 
Elementos fundamentales para el cuidado en guarderías privadas cubanas: perspectiva... $\mid 20$

9. Lacerda MR, Costenaro RGS. Metodologias da pesquisa para a enfermagem e saúde: da teoria à prática [Internet]. Porto Alegre: Moriá; 2016 [acesso em 2018 ago 01]. Disponível em: https://issuu.com/moriaeditoraltda/docs/metodologias_issuu2

10. Bardin L. Análise de conteúdo. São Paulo: Casa de Ideias; 2016.

11. Asociación Médica Mundial (WMA). Declaración de Helsinki de la AMM - Principios éticos para las investigaciones médicas en seres humanos [Internet]. World Medical Association; 2013 [cited 2018 Aug 01]. Available from: https://www.wma.net/es/policies-post/declaracion-de-helsinki-de-la-amm-principioseticos-para-las-investigaciones-medicas-en-seres-humanos/

12. Fonseca TC, Sousa FF, Carballo FP, Fonseca AR, Rabelo DMRS. Fatores associados às enteroparasitoses em crianças usuárias de creches comunitárias. Ciênc Saúde. 2018;11(1):33-40. doi: 10.15448/1983-652X.2018.1.27909

13. Abreu AP, Teston APM, Paula CA, Ribas AD. Epidemiologia das parasitoses intestinais em préescolares e escolares de duas creches em Marialva-PR. IX EPCC - Encontro Internacional de Produção Científica UniCesumar. 2015 nov 03-06; Maringá, PR. Anais. (Braz J Surg Clin Res [Internet]. 2015 [acesso em 2018 ago 01];12(1):4-8. Disponível em: http://rdu.unicesumar.edu.br/handle/123456789/2394).

14. Pedraza DF. Hospitalização por doenças infecciosas, parasitismo e evolução nutricional de crianças atendidas em creches públicas. Ciênc Saúde Colet. 2017;22(12):4105-14. doi: 10.1590/1413812320172212.08212016

15. Soto Lesmes V, Parrado Lozano Y, Gómez Ramírez O, Gomez Ramírez A. Evidence for nursing care in children in healthy environments. Salud Uninorte. 2018;34(2):1-12. doi: 10.14482/sun.34.2.613.04

16. Pedraza DF. Preditores de riscos nutricionais de crianças assistidas em creches em município de porte médio do Brasil. Cad Saúde Colet. 2017;25(1):14-23. doi: 10.1590/1414-462X2017000100xx

17. Pedraza DF, Araujo EMN, Firmino Neta RLB, Oliveira MM, Cunha MAL. Estado nutricional de micronutrientes de crianças segundo características pessoais e das creches. Cad Saúde Colet. 2016;24(4):468-77. doi: 10.1590/1414-462X201600040150

18. Urquiaga Alva ME, Gorritti Siappo C. Estado nutricional y rendimiento académico del escolar. In Crescendo [Internet]. 2012 [citado 2018 ago 01];3(1): 121-9. Disponible en: https://dialnet.unirioja.es/descarga/articulo/5127636.pdf

19. Harton A, Myszkowska-Ryciak J. [The influence of nutrition education on the implementation of the recommendations of daily food ration in selected nurseries-preliminary results]. Stand Med Pediatr [Internet]. 2016 [cited 2018 Aug 01];13: 330-7. Available from: https://www.researchgate.net/profile/Anna_Harton/publication/305211916_Wplyw_edukacji_zywieniowej _na_realizacje_zalecen_modelowej_racji_pokarmowej_w_wybranych_zlobkach_-

_wyniki_wstepne/links/5aa908b34585151788183f76/W plyw-edukacji-zywieniowej-na-realizacje-zalecenmodelowej-racji-pokarmowej-w-wybranych-zlobkach-wyniki-wstepne.pdf

20. Alligood MR. Modelos y teorías en enfermería. 9th ed. Barcelona (ES): Elsevier España; 2018. 
21. Martínez Trujillo N. Metodología para desarrollo de capacidades de investigación en sistemas y servicios de salud en Enfermería. Educ Med Super [Internet]. 2016 ene [citado 2021 abr 08];30(1). Disponible en: http://scielo.sld.cu/scielo.php?script=sci_arttext\&pid=S0864-21412016000100017\&lng=es

22. Páez Patrón JV. Programa de guarderías y estancias infantiles para apoyar a madres trabajadoras. Rev Latinoam Derecho Soc [Internet]. 2007 [citado 2018 ago 0];(5):247-50. Disponible en: https://www.redalyc.org/pdf/4296/429640260013.pdf

23. Ministerio de Educación de la República de Cuba. Educa a tu hijo, programa para crecer con amor [Internet]. La Habana: Ministerio de Educación de la República de Cuba; 2021 [citado 2021 mar 15]. Disponible en: https://www.mined.gob.cu/educa-a-tu-hijo-programa-para-crecer-con-amor/

24. Salbego C, Nietsche EA, Teixeira E, Girardon-Perlini NMO, Wild CF, IIha S. Tecnologias cuidativoeducacionais: um conceito emergente de práxis de enfermeiros em contexto hospitalar. Rev Bras Enferm. 2018;71(Suppl 6):2825-33. doi: 10.1590/0034-7167-2017-0753

25. Enriquez González C, Torres Esperón JM, Alba Pérez LC, Fernández Peñaranda T, Fimia-Duarte R, Crespo Borges T. Tecnologías educativas dirigidas a cuidadores de infantes con trastorno por déficit de atención e hiperactividad. Biologist [Internet]. 2020 [citado 2020 jun 30];18(1):155-60. Disponible en: http://revistas.unfv.edu.pe/index.php/rtb/article/download/609/543

26. Pérez Adame E, Fulgencio Juárez M, González Zepeda AP. Burnout en personal de estancias infantiles y su relación con las habilidades y conocimientos requeridos para el puesto. Rev Educ Desarro [Internet]. $2013 \quad$ [citado 2018 ago 01];24:5-12. Disponible en: https://www.cucs.udg.mx/revistas/edu_desarrollo/anteriores/24/024_Perez.pdf

27. United Nations Children's Fund (UNICEF). Para cada niño, reimaginemos un mundo mejor: informe anual de UNICEF 2019 [Internet]. New York: UNICEF; 2020 [citado 2021 feb 15]. Disponible en: https://www.unicef.org/media/71156/file/UNICEF-informe-anual-2019.pdf

28. Cerqueira ACDR, Cardoso MVLML, Viana TRF, Lopes MMC. Integrative literature review: sleep patterns in infants attending nurseries. Rev Bras Enferm. 2018;71(2):424-30. doi: 10.1590/0034-7167-2016-0480

29. Oropeza-Bahena G, López-Sánchez JD, Granados-Ramos DE. Hábitos de sueño, memoria y atención en niños escolares. Rev Mex Neuroci. 2019;20(1):42-9. doi: 10.24875/RMN.M19000021

30. Wajskop G. Linguagem oral e brincadeira letrada nas creches. Educ Real. 2017;42(4):1355-74. doi: $10.1590 / 2175-623661980$ 
Elementos fundamentales para el cuidado en guarderías privadas cubanas: perspectiva... | 22

Editor Científico jefe: Cristiane Cardoso de Paula

Editor asociado: Aline Cammarano Ribeiro

Promoción / Reconocimiento: a las enfermeras participantes en los grupos focales y a las investigadoras alternas.

\section{Autor correspondiente}

José Martín Medina Pérez

E-mail: jmmperez@infomed.sld.cu

Enderezo: Calle Candelaria, No. 192 entre Alemán y Carretera Central. Santa Clara, Villa Clara, Cuba. CEP: 54500

\section{Contribuições de Autoria}

\section{1 - José Martín Medina Pérez}

Concepción o diseño del estudio/investigación, análisis y/o interpretación de datos, revisión final con participación crítica e intelectual en el manuscrito.

\section{2 - Julia Maricela Torres Esperón}

Concepción o diseño del estudio/investigación, análisis y/o interpretación de datos, revisión final con participación crítica e intelectual en el manuscrito.

\section{3 - Carilaudy Enriquez González}

Análisis y/o interpretación de datos, revisión final con participación crítica e intelectual en el manuscrito.

\section{Cómo citar este artículo}

Pérez JMM, Esperón JMT, González CE. Fundamental elements for the care in Cuban private nurseries: a Nursing perspective. Rev. Enferm. UFSM. 2021 [Accessed on: Year Month Day]; vol.11 e57: 1-22. DOI: https://doi.org/10.5902/2179769265081 